\title{
A Unique Approach to Visualising Sustainability in the Built Environment
}

\author{
Mr John Isaacs \\ Whitespace Research \\ University of Abertay Dundee \\ j.Isaacs@abertay.ac.uk
}

\author{
Dr Ruth Falconer \\ School of Contemporary \\ Sciences \\ University of Abertay Dundee \\ r.Falconer@abertay.ac,uk
}

\author{
Dr David Blackwood \\ Urban Water Technology \\ Centre \\ University of Abertay Dundee \\ d.Blackwood@abertay.ac.uk
}

\begin{abstract}
Developing sustainable urban environments is complex as it requires consideration of interacting social, economic and environmental sustainability factors. The task is made even more difficult by the wide variety of stakeholders (e.g. planners, architects, businesses and the public) that may be involved in the process and the lack of a common language for all to understand. This paper describes a prototype visualisation tool (S-City VT) that will allow stakeholders to understand and influence decisions regarding the sustainability of an urban development. $S$-City VT models the interactions between the sustainability indicators, using the analytical network process (ANP) methodology. The results of the ANP model are shown to the user in a novel way using a $3 D$ visualisation tool. The user will be presented with a $3 D$ visualization of the 'virtual' development that encapsulates the results of the ANP model and thus the sustainability of the virtual development. By taking this approach S-City VT provides a common language for the theory of sustainability which will be understood by all stakeholders. Further, the tool will allow evaluation of the consequences of decisions relating to the short and long term implications of alternative courses of action.
\end{abstract}

\section{Background}

\subsection{The need for sustainable cities}

The world, especially the developed countries, has become increasing dependant on its urban centres that contain the overlapping communication, trade and production networks. It is these urban centres that provide the basis for a nation's development by controlling the flow of information, energy, commerce and people [2]. In 1950 the proportion of the world's population that lived in urban areas was just under $30 \%$, by 2005 the proportion had risen to $47 \%$. The
United Nations predict that this trend of urbanisation will continue into the future [16].

Our cities continue to grow both economically and physically to provide the growing number of citizens with the way of life they aspire to. This increase in city population and size increases the pressure on the natural resources and can also reduce living standards as the resources that are available have to be spread over a larger number of people and over a larger physical area. There is no upper limit to physical city size, population or resource use at which an ecological disaster will occur. It is extremely unlikely that once a certain value is reached that a sudden disaster will manifest, it is however much more likely that once the threshold has been crossed that gradual rise in costs and diminished returns will be seen [2].

\subsection{Sustainable development}

In the urban context a sustainable development is one that meets the needs of the present population without compromising the needs of the next generation. It is the aim of sustainable urban development to provide "more effective and efficient services, that maintain public health and welfare, whilst reducing harmful resource and environmental impacts" [6].

Incorporating sustainability in urban development is extremely complex as it requires the consideration of the social, economic and environmental impacts on the city or area being developed (Figure 1). 


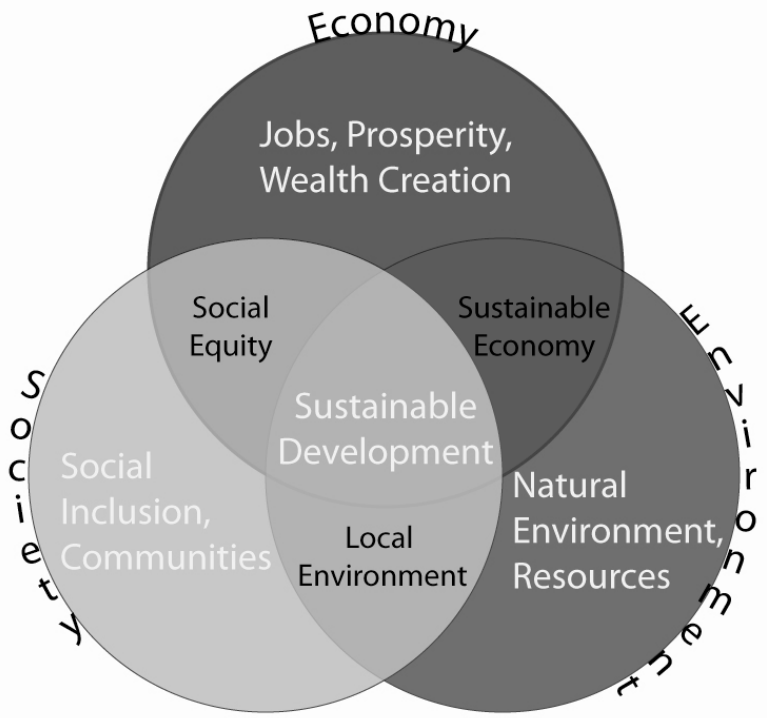

Figure 1: Sustainability factors

Sustainability can be measured using principles, criteria and indicators [3]. Principles are usually abstract, idealistic statements that provide goals in order for sustainability to be achieved. Criteria are a set of factors that can be used to make a judgment about the relative sustainability of a number of options or scenarios. Indicators are measurable past and present values for specific criteria and can also be used to set standards against which future performance can be assessed [6].

\subsection{Decision support tools}

A decision support tool (DST) is simply a tool that is used to aid decision making as part of a formal or informal decision-support process. The use of DSTs has become increasingly more popular, mainly because it is possible to install and use them on personal computers and also due to their ability to manage large amounts of complex data [9].

A number of decision support tools have been created to aid decision makers in achieving sustainable urban developments, which in itself is a complex task. There has been huge effort and resources put into creating DSTs, yet despite this most are never or hardly ever used [15].

\subsection{Failure of existing tools}

The main reason the tools are rarely used is due to a combination of factors. Many are too complicated to be used by anyone but an expert, and require specialised training. There may be a lack of trust between the user and the results, the so called black box effect. The output from the tool is extremely important in determining whether the tool will be used or not, in most existing tools it is either too little to be useful or too complicated to be accessible [15].

\section{The need for 3-dimensions}

When 3-dimensional visualisation techniques are used correctly to explain complex systems they can hide the underlying complexity while providing the viewer with a simple and accessible representation [17]. This is also true in the case of sustainability assessment where a good decision support tool should include " three-dimensional visualisation, virtual reality, animation and other advanced computerised visualisation techniques" [9]. A tool using 3dimensional visualisation will help the user determine the effect of alternative courses of action on the sustainability of the urban development, without having to understand the complex interactions between the various aspects.

A 3-dimensional visualisation tool will offer a more realistic view of the environment, by incorporating concepts from computer game technology that aim to achieve realistic game worlds. This will be an advantage over 2-dimensional approaches and will model the development more accurately, as many cities in the world today have become multi layered, with each level of a building providing a vastly different service to the city that the levels below or above it. For example, the ground floor of a building can contain a restaurant or shop, the 1st floor a solicitor's office, while the remaining floors could house residential apartments. Each of these separate levels will have a different effect on the sustainability, and this cannot be accurately shown on a 2D representation (Figures $2 \& 3$ ). The most obvious advantage of a 3D representation of an urban development is its ability to allow future developments to come to life using animations relating to the alternative courses of action. 


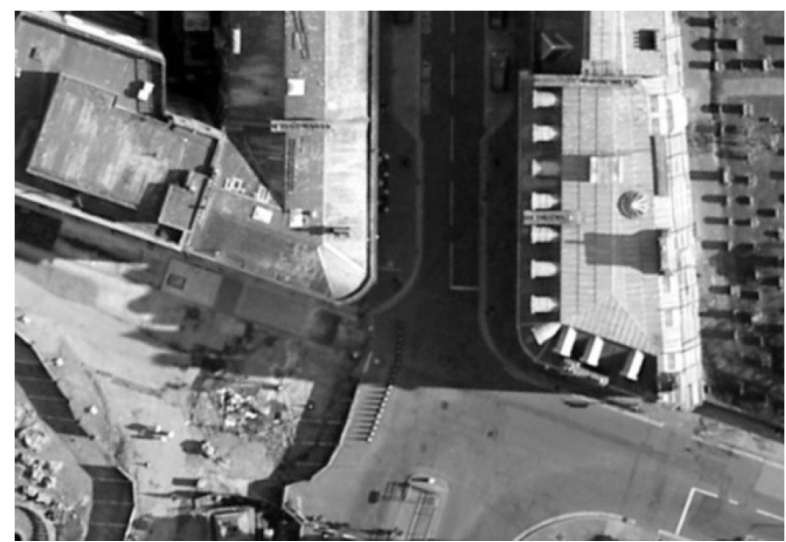

Figure 2: 2-Dimensional view of an urban environment. [7]

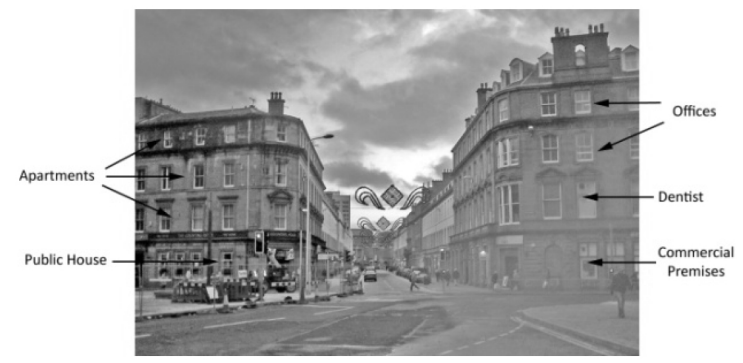

Figure 3: 3-Dimensional view of the same urban environment

\section{S-City VT}

The Sustainable City Visualisation Tool (S-City VT) has been designed to address the lack of visualisation techniques available in existing sustainability decision support tools. Currently there are three main interfaces which allow the user to access the functionality of the application.

\subsection{Model interface}

S-City VT uses the Analytical Network Process (ANP) to model the complex interactions involved in sustainability. The ANP method uses interactive network structures that give a holistic representation of the overall problem [14]. To perform ANP analysis the decision maker must identify the network through analysis of the problem to be solved. The decision maker must identify the clusters, elements, and the relationships and interactions among them [1]. An example network for a sustainable development scenario is shown in Figure 4.

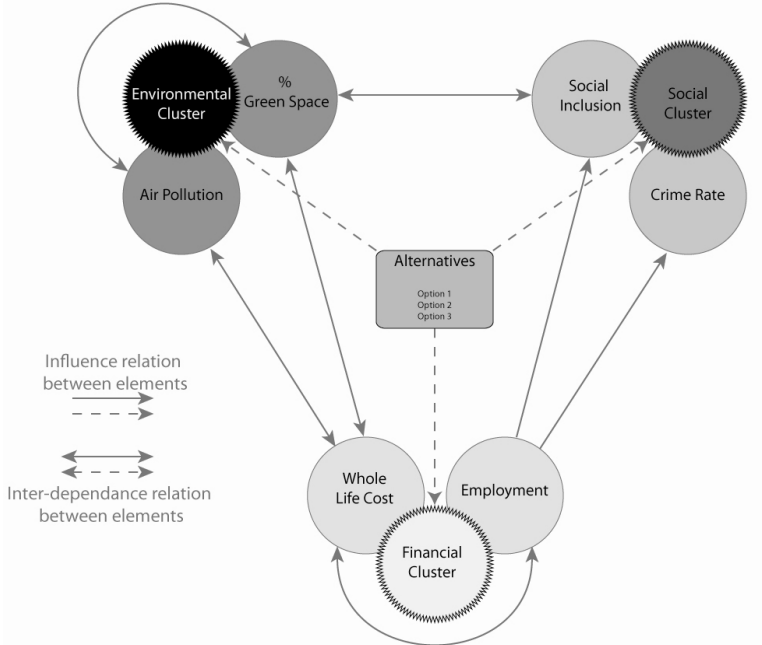

Figure 4: Example sustainable development network model.

In the S-City VT application the network used by the ANP model will be defined by the users opinions regarding the influence of each indicator (e.g. air pollution, social inclusion or population) on other indicators in the development.

The model interface allows the user to input opinions that will be used by the application to generate the network required for the ANP model. The user is presented with a matrix that represents a specific cluster or indicator in the network. The user can subsequently enter their views on how important the influence of one indicator or cluster is over another. This creates a system of pair-wise comparisons. The comparison matrix for the environmental cluster is shown in Figure 5.

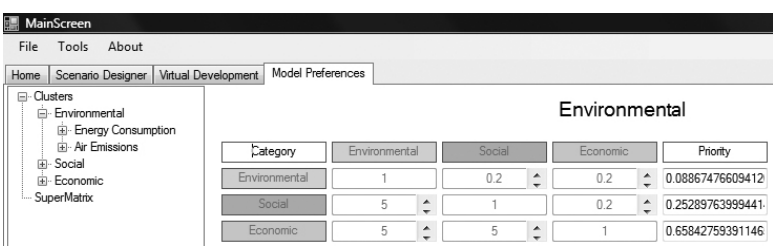

Figure 5: Comparison matrix screen for the environmental cluster

Once the user has expressed their views using the pairwise comparisons, the ANP model generates a supermatrix describing every interaction between the indicators in terms of every other indicator it interacts with [13]. 


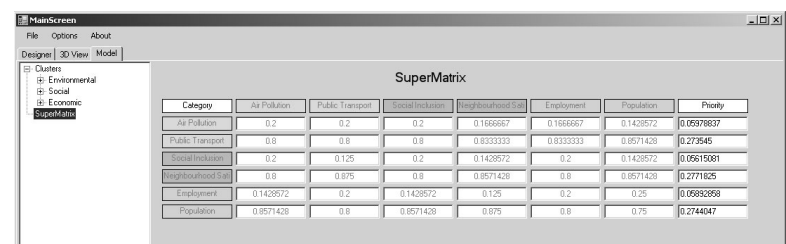

Figure 6: Example Supermatrix

By describing the interactions between the indicators, the supermatrix defines the network used by the ANP model. The ANP model uses this network to calculate the priority of the clusters and indicators, enabling the indicator values to be weighted correctly for the visualisation.

For an urban environment to be truly sustainable all people of need, interest, experience and geographical proximity to a development should be involved in its creation and maintenance [8]. The use of ANP allows all stakeholder's opinions to be considered. This is extremely important different stakeholders will place different emphasis on specific sustainability indicators, therefore a scenario that is viewed as sustainable to one stakeholder may be viewed as unsustainable by another. It is also the case, due to the lengthy life cycle of urban developments that opinions about the importance of specific indicators may change as future communities realise what is important to them.

\subsection{Design interface}

The design interface allows the user to create a 2D grid-based view of the development. It is primarily designed to enable the planner's $2 \mathrm{D}$ plans to be easily translated into the application.

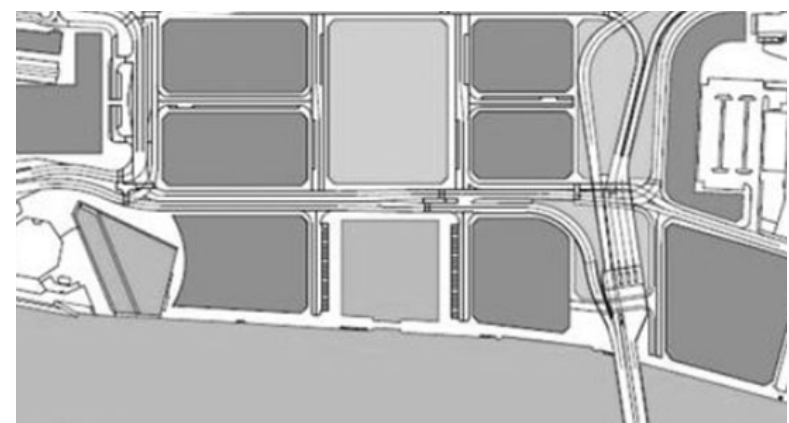

Figure 7: 2D Development plan [4]

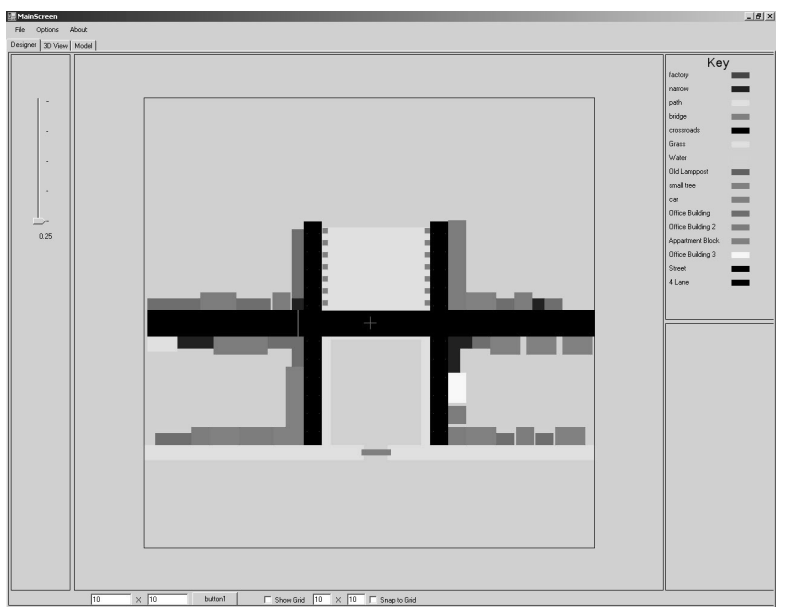

Figure 8: S-City VT design screen showing development in Figure 7.

Components such as buildings, streets, lamp-posts, trees, water and grass, can be added and positioned on the design screen. All the possible structures for a specific development are stored in a database within the application. The database of structures contains 3D models created by the planners or architects involved in the development and can be updated or changed at anytime.

The designer takes the footprint of the 3D model and displays this on the design panel. This enables the planner to systematically build up the complete $2 \mathrm{D}$ plan of the development.

The design screen also allows the user to select a building and assign it properties that will affect its sustainability. Values for sustainability indicators such as air pollution, neighbourhood satisfaction or capital cost can all be entered for the specific building or structure.

Once the design has been created, the user may return to the design screen and changes to the design will be updated dynamically. Any changes made will immediately be reflected in the ANP model and also the $3 \mathrm{D}$ view, allowing the user to see the consequences of their decision and to determine the effects of different scenarios.

\subsection{D interface}

The 3D interface of the application is the main visualisation component of the application. The 3D view allows the user to visualise the development, based on the 2D plan created in the design interface. 


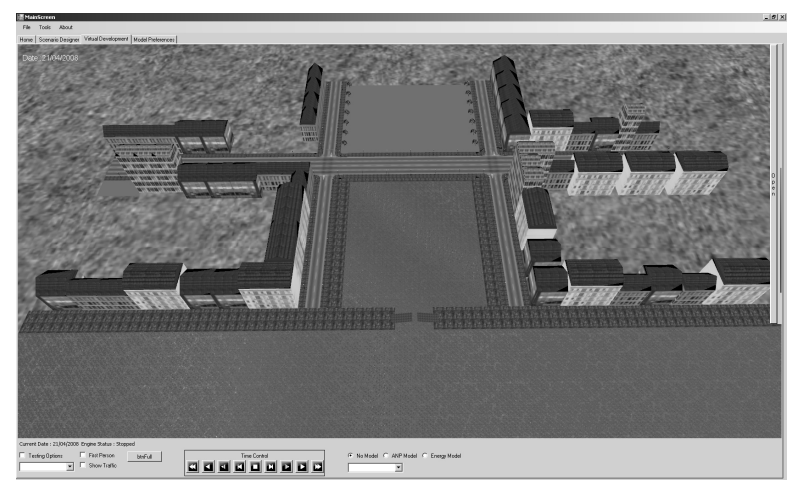

Figure 9: 3D view of development created in Figure 8.

The 3D interface makes use of a custom 3D engine, allowing seamless integration of the modelling and visualization components required to determine the sustainability of alternative development scenarios. Using the 3D interface the user is able to view the development, from first person and birds eye views, enabling the user to envisage the development from any viewpoint they wish (Figure 10).

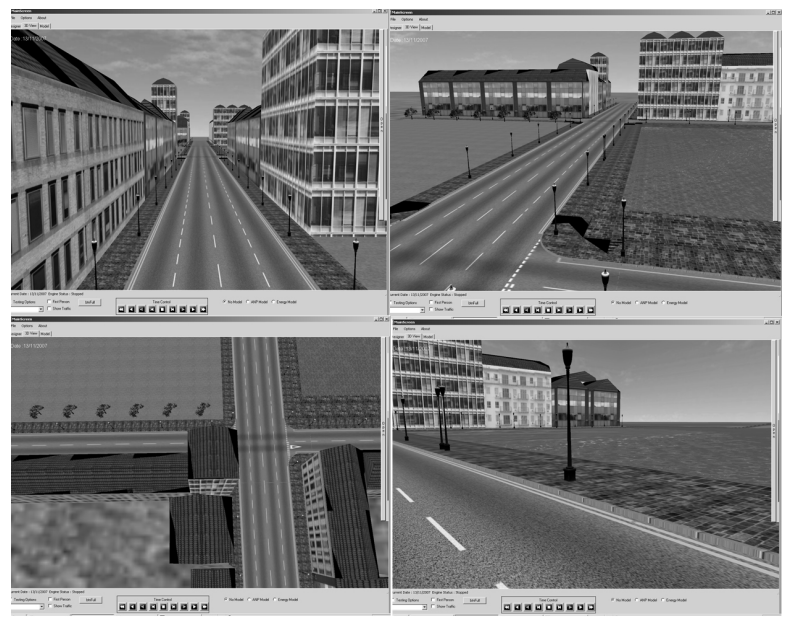

Figure 10: 3D view flexibility

It has been shown [11] that users will interact with animated visualisations to identify when data values change prominently, to find values at particular points in time and to acquire a feeling of immersion in order to more intuitively understand the data being presented. S-City VT fulfils this by using its 3Dimensional visualisation to display the results of the ANP model, demonstrating the sustainability of the urban development and its components. The sustainability is a result of real indicator data from the development i.e. values for $\mathrm{CO}_{2}$ emissions, and is combined with the weightings supplied by the ANP model.

\section{Model results}

This framework allows users to ask not only "what if ..." questions in relation to the urban development, i.e. for a prescribed input what is the predicted output, but also the reverse query "if ... what", i.e. for a target end state (output) what is the required parameter set (input). The tool will allow the user to investigate how sustainable different developments will be. Sustainability is shown by blending several colour maps and there is a colour map for each indicator used.

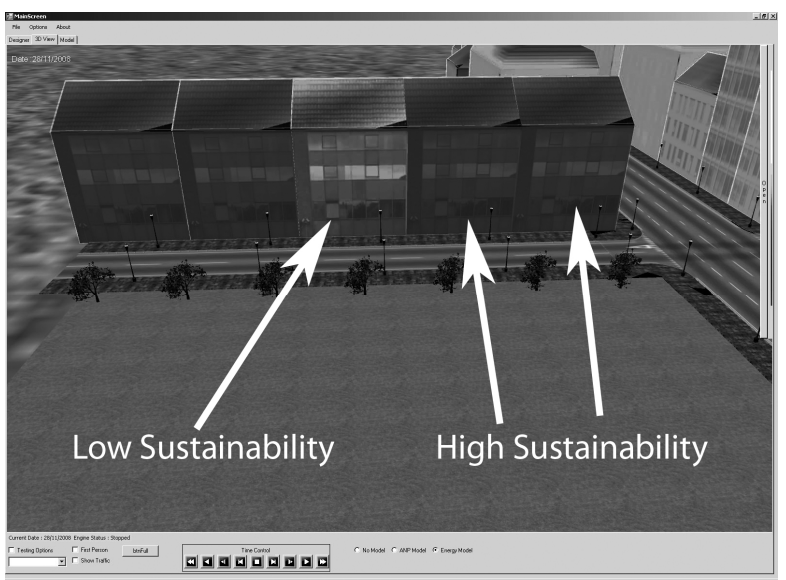

Figure 11: Original colour system showing energy use per building.

This layering approach works well when only a small number of indicators are used or when comparing two extreme values, as shown in Figure 11. However it was discovered, via preliminary usability testing, that very large changes in a single indicator would not result in a significant change in the final blended colour.

The colour mapping was extended and incorporates the results from ANP model to provide the relative weighting of the indicators. The final colour displayed on the visualisation tool is determined by calculating a weighted, normalised value for each indicator, this is subsequently mapped to a colour scale. Due to the use of the ANP weightings, the importance of the indicator will be predefined and a small change will not be 'washed out' by indicators that the user has defied as less important. This is shown in Figure12, where the cumulative results of 6 indicators (air pollution, public transport use, social inclusion, neighbourhood satisfaction, employment and population). 


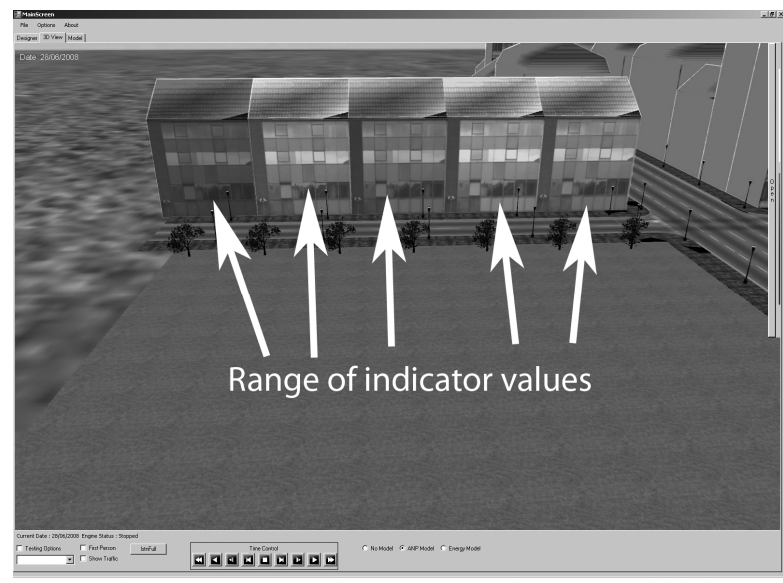

Figure 12: New colour system showing a range of indicators

The 3D interface includes a time control, similar to a media player control, with play, pause, stop etc. The time control allows the user to view the sustainability of the overall development or individual buildings at any point in the future or 'rewind' time to analyse a specific time point using the time control. Further by clicking on a building more detailed information relating to its sustainability can be obtained via sustainability triangle (Figure. 13).

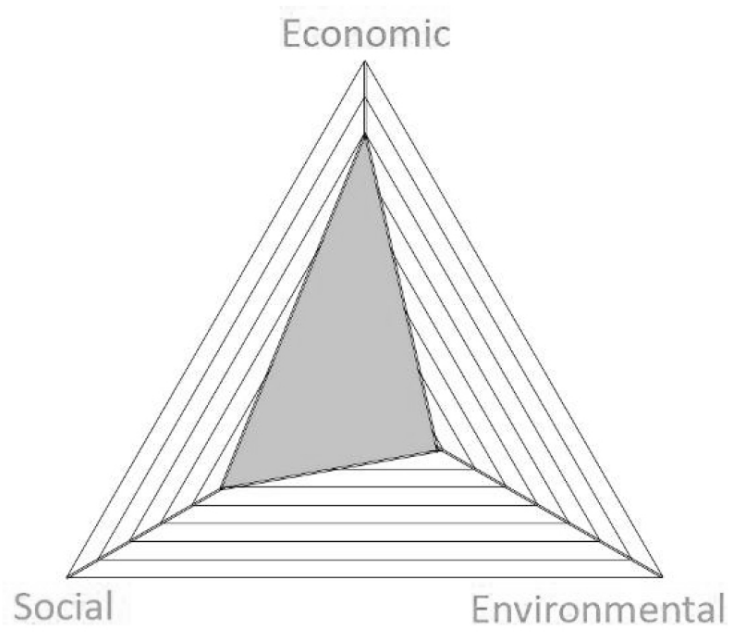

Figure 13: Radar graph showing detailed sustainability information

\section{Other visualisation techniques}

Colour overlays are one method of conveying the sustainability of the development. There are some indicators that can be described in a more intuitive way. For example traffic simulation is used to give the user a representation of how different traffic densities affect the sustainability of the development. Currently the number of cars is simply an arbitrary representation of traffic density.

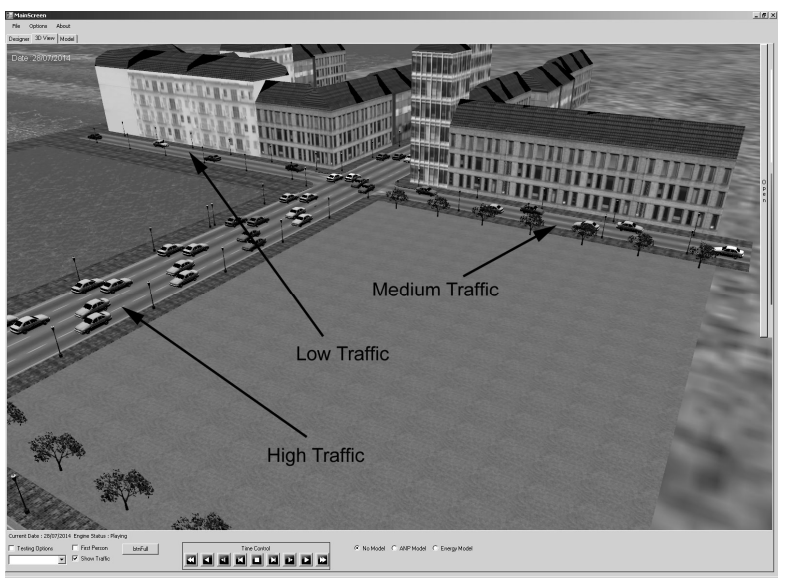

\section{Figure 14: Simulating different levels of traffic density}

The S-City VT 3D engine has also been developed to allow the inclusion of directional sound effects. The inclusion of sound allows S-City VT to become a truly immersive experience adding another layer of realism. Currently traffic sound effects are being used to illustrate traffic densities. Sound effects, such as bird song, can also be used, in the representation of public spaces, such as inner city parks to help show the effect of public spaces on the ambient urban noise.

\section{Future work}

There are many ways in which the tool can be extended. Currently S-City VT uses a simple traffic simulation to represent the traffic density indicator. However it is envisaged that more realistic techniques will be added to the visualization.

Crowd modelling can show how the population will move through the development. The crowd movement emerges as a consequence of interactions with other individuals in the crowd and with the environment [12]. Crowd modelling will be useful in determining the crowds response to specific scenarios, in the proposed development, and can be used to investigate if natural movement or bottle necks will occur [5]. Social aspects can be illustrated by the crowd. For example in an area of high neighbourhood satisfaction the individuals could walk in a way that shows happiness, waving hands cheering etc. An area with low satisfaction can be represented by sad people, with heads hanging down and shuffling of feet. For a crowd with mixed happiness i.e. individuals displaying both 
sad and happy behaviour the individuals could appear indifferent or normal. The crowds emotional state will directly affect the crowds behaviour, where emotional groups of individuals will adhere to differ sets of rules depending on the emotional state (i.e. level of happiness or sadness), and will affect how they are perceived by the user [10].

Finally, to make the visualization more realistic and immersive particle effects representing pollution and related to traffic density can be incorporated into visualization. Animated sprites could also be used to represent waste generation, where a very wasteful area would have overflowing bins or litter lying in the streets. These techniques and more like them can all be used to better engage the stakeholders in the planning process.

To determine the effectiveness of the S-City VT tool a number of specific scenarios will be developed based on the waterfront regeneration development in Dundee. A usability study will assess if scenarios, such as rapid population increase or a dramatic change in housing provision can convey the relative sustainability of the development to a range of stakeholders .

\section{Hive}

While S-City VT can be executed on most modern personal computers, it has also been designed in conjunction with the HIVE (Human Immersive Virtual Environment) facility at the University of Abertay. The HIVE facility is designed to create a completely immersive environment through the use of 3-D stereo projection, surround sound, data gloves and tracking systems. The facility enables a large number of individuals to become immersed in the environment being created, while their interactions with the environment can be observed from a separate control room. For S-City VT this enables a large number of stakeholders to participate in planning decisions simultaneously, while being surrounded by the virtual development they are creating.

\section{Conclusion}

It is clear that decision support tools have an important role to play in the creation of sustainable urban environments. Existing tools however are still largely only accessible by expert stakeholders involved in the planning process. Even with their use by experts, existing tools are only capable of describing small parts of a development, transport for example, or a single feature, such as the environment.
It is evident that the complexity of urban sustainability and the current methods used to assess it are a barrier to engaging more stakeholders in the planning process. The exclusion of any stakeholders from the planning process can lead to these individuals or groups becoming disillusioned with the development as they will not feel as if they have played a part in its creation. To address this issue it is extremely important that the urban planning process, particularly sustainability assessment, be as open, transparent and available to as many stakeholders as possible.

The S-City VT application hopes to address the drawbacks of the current decision support tools by combining an integrated simulation model (ANP) with a visualisation tool. The creation of a $3 \mathrm{D}$ virtual environment allows a stakeholder to feel much more a part of the development because they can actually see it come to life. By projecting the results of the simulation model onto a virtual representation of the actual development, S-City VT allows the user to immediately envisage the consequences of any decisions that they make, and the differences in specific scenarios, over a number of years.

Obviously sustainability assessment would be much easier if sustainability could be measured using a single indicator. There is currently much discussion about the use of a single sustainability indicator by combining the indicators in a variety of ways. S-City VT takes a different approach and will provide a number of methods i.e. single blended combined indicator value and novel techniques of preserving individual indicator values to convey the complex sustainability information to a variety of stakeholders. The application allows a user to "see" which of the possible scenarios, is in their opinion more sustainable. The use of visualisation techniques, as presented in this paper, begins to remove sustainability assessment's reliance on the existing expert systems, which are largely inaccessible to many of the stakeholder groups, especially the general public.

Note: Colour images and videos of the prototype application are available from www.scityvt.co.uk.

\section{References}

[1] Bottero, M., Modini, G. and Valle, M. 2007. The use of the Analytic Network Process for the sustainability assessment of an urban transformation project: 07. In: Horner, M., et al. eds. International Conference on Whole Life Urban Sustainability and its Assessment: M, Glasgow, BMG pp.209 - 231. 
[2] Brundtland, G. H. 1987. Our Common Future/World Commission on Environment and Development. Oxford University Press.

[3] Defra. 2005. Securing the future : delivering UK sustainable development strategy. [London]: The Stationary Office.

[4] Dundee City Council Dundee Central Waterfront Development Masterplan 2001 - 2031 Planning \& Transportation Department.

[5] Farenc, N., et al. 2000. A Paradign for Controlling Virtual Humans in Urban Environment Simulations: 00. Applied Artificial Intelligence. 14(1): pp.69-91.

[6] Foxon, T. 2002. Sustainability criteria for decision support in the UK water industry.: 02. Journal of Environmental Planning and Management. 45(2): pp.285301.

[7] GeoInformation Group. 2007. Google Earth Images.

[8] Haughton, G. and Hunter, C. 2003. Sustainable Cities. Routledge.

[9] Kapelan, Z., Savic, D. and Walters, G. 2005. Decisionsupport tools for sustainable urban development: 05 . Engineering Sustainability. 158(3): pp.135 - 142.

[10] Musse, S. R. and Thalmann, D. 2001. Hierarchical model for real time simulation of virtual human crowds: 01 .
IEEE TRANSACTIONS ON VISUALIZATION AND COMPUTER GRAPHICS. 7(2): pp.152-164.

[11] Nakakoji, K., Takashima, A. and Yamamoto, Y. 2001. Cognitive effects of animated visualization in exploratory visualdata analysis: 01. Information Visualisation, 2001. Proceedings. Fifth International Conference on. pp.77-84.

[12] Pelechano, N., et al. 2005. Crowd simulation incorporating agent psychological models, roles and communication: 05. First International Workshop on Crowd Simulation.

[13] Saaty, T. L. 1999. Fundamentals of the analytic network process: 99. Proceedings of International Symposium on Analytical Hierarchy Process.

[14] Saaty, T. L. ed. 2006. The Analytic Network Process. Melbourne: Idea Group Inc.

[15] Sahota, P. S. and Jeffrey, P. 2005. Decision-support tools: moving beyond a technical orientation: 05 . Proceedings of the Institution of Civil Engineers, Engineering Sustainability. 158(ES3): pp.127-134.

[16] UNDESA World Urbanization Prospects: The 2005 Revision United Nations: Department of Economic \& Social Affairs

[17] Young, P. 1996. Three Dimensional Information Visualisation: 96. Computer Science Technical Report. 12 p.96. 